\section{EL PANDEO EN LA INSTRUCCION EH-80}

\author{
Francisco Morán Cabré y \\ Hugo Corres Perretti, \\ Drs. Ingenieros de Caminos
}

$400-7$

\section{Introducción. Antecedentes}

Desde la aparición, hace ya ocho años, de la Instrucción EH-73 y principalmente con motivo de los coloquios que se suscitaron durante los Cursillos sobre la misma, organizados por la Comisión Permanente del Hormigón, y cuya conferencia sobre Pandeo estuvo a cargo del primer autor, se ha tenido ocasión de recoger numerosas criticas de los proyectistas españoles al articulado sobre pandeo contenido en dicha Instrucción. Estas criticas han puesto de manifiesto las deficiencias de aquel articulado, tanto en términos absolutos como en comparación con los de las Normas de Hormigón Armado de otros paises.

En efecto, y para mencionar sólo algunos puntos, la Instrucción EH-73:

- consideraba únicamente los fenómenos de segundo orden en piezas, pero no en pórticos (pandeo global o efecto $P-\Delta$ );

- definia longitudes de pandeo que, para el caso más frecuente de pórticos de edificación, eran claramente inferiores a las reales, lo cual iba en contra de la seguridad en muchos casos (soportes de pórticos traslacionales);

- asimilaba los efectos de segundo orden a la consideración de una excentricidad adicional $e_{a}$ definida mediante una fórmula aproximada, pero no limitaba el campo de validez de esta fórmula para grandes esbelteces, para las que sus resultados no eran buenos;

- por otra parte, la fórmula había sido deducida para el caso más desfavorable del soporte con excentricidades iguales en sus extremos, por lo cual en el caso más frecuente de soportes de edificación, con excentricidades de distinto signo, conducia muchas veces a resultados demasiado conservadores;

- por último, la fórmula correspondia al caso de pieza de sección y armadura uniformes (aunque esto no se decia explicitamente), por 10 cual quedaba sin cubrir el caso de piezas de sección o de armado variables.

A la vista de este deficiente tratamiento del pan deo, la Comisión Permanente del Hormigón decidió revisar el articulado correspondiente con motivo de la preparación de la Instrucción EH-80, a cuyo efecto dispuso la creación de un Grupo de Trabajo de Pandeo, del cual formaron parte los señores Garcia Meseguer (del Instituto Eduardo Torroja, ponente en la Comisión Permanente del Hormigón), Agueda (INTECSA), Corres (Instituto Eduardo Torroja, ponente en el Grupo de Trabajo), Chueca (José Antonio Torroja Oficina Técnica), Fernández Sánchez (Instituto Eduardo Torroja), Llombart (Caminos y Puertos), Morán (Instituto Eduardo Torroja) y Sáinz Román (Sereland). Dicho Grupo trabajó desde junio de 1977 hasta diciembre de 1978, y sus principales actividades fueron las siguientes:

- estudio del tratamiento del pandeo en las principales Normas de Hormigón Armado, especialmente en las más recientes (CEB Model Code 78, ACl 318-77, CP 110-72 y DIN 1045-72);

- análisis comparativo de los resultados de los distintos métodos aproximados propuestos por estas normas;

- elaboración de una primera propuesta de articulado sobre pandeo para la futura Instrucción EH-80;

- celebración de una Mesa Redonda sobre Pandeo, que tuvo lugar en el Instituto Eduardo Torroja el 29 de noviembre de 1978, con asistencia de quince especialistas, y en la que se expuso dicha propuesta y se recibieron comentarios y sugerencias sobre la misma;

- preparación de una propuesta de articulado sobre pandeo, que fue sometida por el ponente García Meseguer a la Comisión Permanente del Hormigón, y que ha constituido la base del actual articulado sobre el tema en la Instrucción EH-80.

\section{Longitud de pandeo en piezas de estructuras intraslacionales y traslacionales}

Como es sabido, el cálculo en segundo orden de una pieza comprimida, tanto si es aislada como si forma parte de un pórtico, se aborda considerando 
52

Informes de la Construcción/331

la pleza biarticulada de sección constante equivam lente. El primer problema consiste pues en la obtención de la longitud de esta pleza, o longitud de pandeo Io de la pieza primitiva. Suele suponerse que para esta longitud puede tomarse la distancia entre los puntos de inflexión, o de momento flec. tor nulo, de la deformada o ligura de pandeo de la pieza primitiva. Es por consiguiente necesario estudiar cuál será esa figura, lo cual depende de las vinculaciones en los apoyos de la pleza, si es aislada, o de las caracteristicas elásticas del pórtico, sl forma parte de uno.

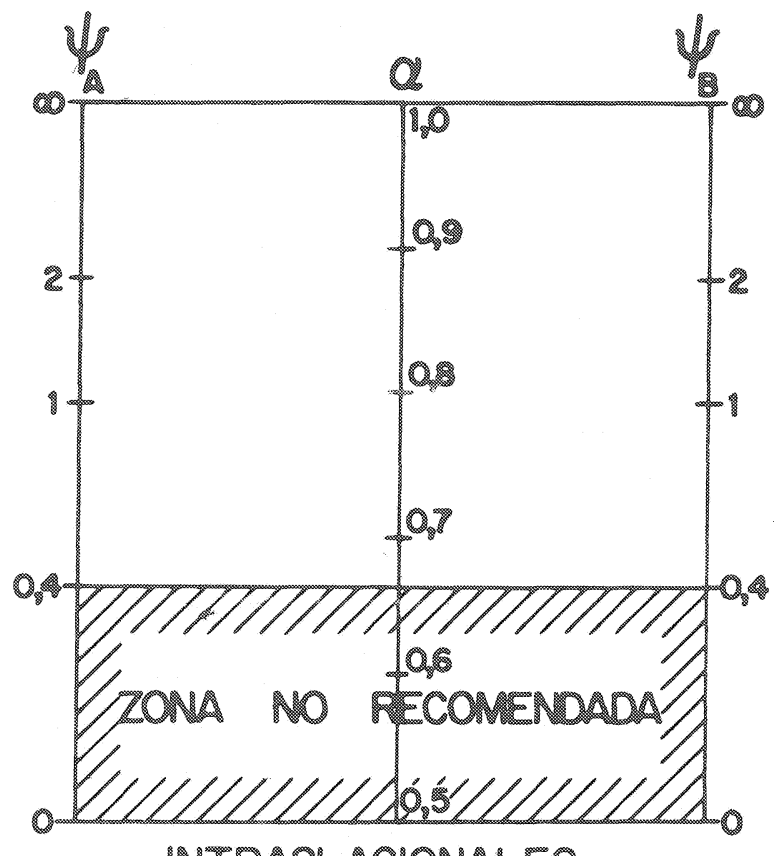

INTRASLACIONALES

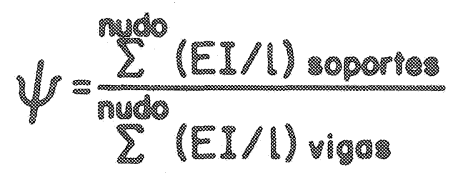

Su aplicación a pórticos reales de hormigón armado en el rango no lineal proporciona únicamente resultados aproximados.

b) Se presentan por separado los casos de pórticos intraslacionales y traslacionales, con re sultados muy distintos, como cabia esperar de la fuerte influencia de los desplazamientos horizontales en la figura de pandeo.

La primera e importante decisión del proyeclista debe consistir en clasificar su pórtico en

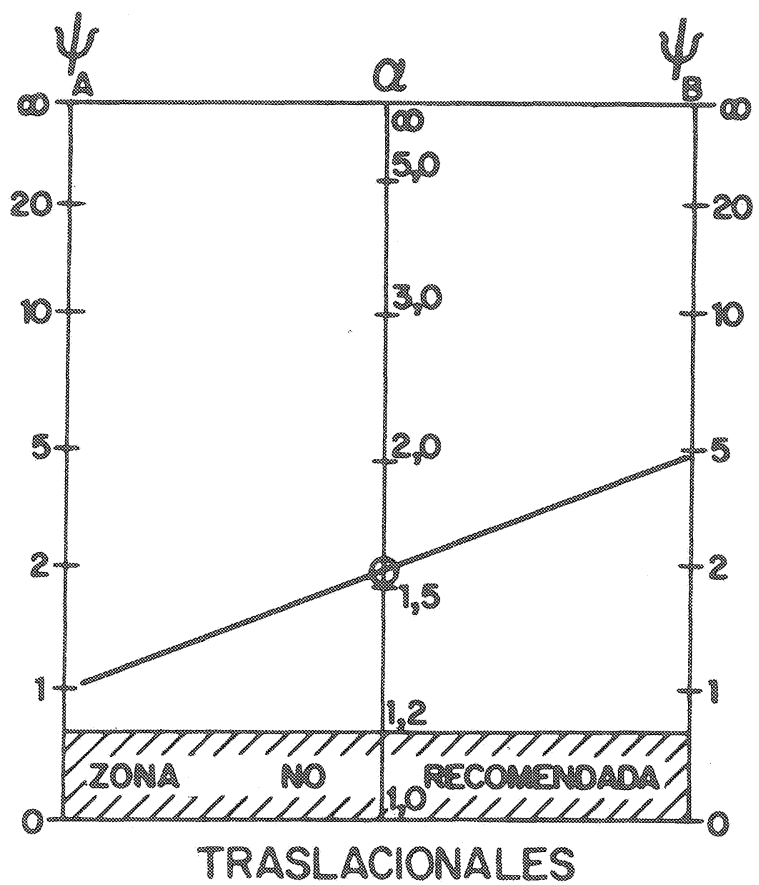

$$
1_{0}=a \cdot 1
$$

Fig. 1. - Nomogramas para la obtención de la longitud de pandeo de soportes en pórticos.

Para la obtención de las longitudes de pandeo de soportes de pórticos regulares de ediricación la EH-80, en el comentario al articulo 43.1 .2 , presenta los nomogramas de la figura 1 , basados en Trabajos de Jackson y Moreland (1), (2). La longl rud de pandeo se obtiene multiplicando la longl tud del soporte por el factor a dado por el nomo grama en función de las rigideces relativas de las vigas y soportes que concurren en los nudos extremos del soporte considerado.

Sobre el uso de estos nomogramas pueden hacer se las siguientes observaciones:

a) Los nomogramas han sido preparados considerando las figuras de pandeo de soportes per tenecientes a pórticos regulares (iguales luces, alturas e inercias) y de gran número de pisos y vanos, en comportamiento elástico y lineal. una u otra categoria. En realidad es muy difi. cil establecer unas reglas sencllas que permi. tan clasificar un pórtico como rraslacional o intraslacional. La idea que debe lenerse en mente sue en pónticos traslacionales, debi. do a los desplazamientos horizontales, la figu. ra de pandeo se modifica por completo res. pecto al caso de los pórticos intraslacionales, dando origen a una longilud de pandeo mayor que la del soporte.

Para pónicos somefidos sólo a cargas vertica. les, a pesar de la pequeña rigidez lateral que pudieran presentar, parece lógico utilizar lon. gifudes de pandeo correspondientes al ábaco de pónticos intraslacionales. Para pórticos so. metidos a cargas horizontales, la magnilud de las mismas Irente a su rigidez lateral dará un indice del grado de deformabilidad del mismo que indicară a ábaco a considerar. 
En este sentido, la Instrucción EH-80 presenta, en el comentario al articulo 43.3 , un crite rio de intraslacionalidad expresado mediante una formula senclla. Debe decirse que este criterio, tomado de la DIN 1045-72 y del CEB Model Code 78, es muy exigente, de forma que resulta imposible cumplirlo salvo que los pórticos vayan arriostrados en las dos direcclones por pantallas o núcleos rigidizadores de dimensiones importantes.

Según el Código norteamericano $\mathrm{ACl} 318-77$ (3) una estructura puede considerarse intrasla cional, a nivel de un piso determinado, si la suma de las rigideces de los elementos arriosiradores (pantallas, núcleos, etc.) es no menor que seis veces la suma de las rigideces de todos los soportes en ese piso. Esta condim ción es bastante menos exigente que la anterior.

De cualquier forma parece claro que las limitaciones impuestas por las fórmulas de las distintas normas se refieren al caso de pórticos con cargas horizontales de una cierta en tidad, ya que sólo tienen en cuenta las condiciones de rigidez lateral de los mismos.

c) Aunque en la EH-80 no se limitan los valores de los factores $\psi$ (relaciones de rigidez), tan to en el Código ACl 318-77 como en la Norma DIN 1045-72 se limitan inferiormente dichos valores, recomendándose que no se utllicen los que quedan en las zonas rayadas de la tim gura 1, incluso si se trata de soportes empo trados en la cimentación.

d) En lugar de los nomogramas para la obtenclón de la longitud de pandeo pueden emplearse las siguientes fórmulas, que son equilentes a los mismos (1):

- para porticos intraslacionales:

$\alpha=\frac{0,64+1,4\left(\psi_{A}+\psi_{B}\right)+3 \psi_{A} \psi_{B}}{1,28+2\left(\psi_{A}+\psi_{B}\right)+3 \psi_{A} \psi_{B}}$

- para pórticos traslacionales:

$\alpha=\sqrt{\frac{7.5+4\left(\psi_{A}+\psi_{B}\right)+1,6 \psi_{A} \psi_{B}}{7.5+\left(\psi_{A}+\psi_{B}\right)}}$

e) Leonhardt (4) recomienda, en el caso de pórt. cos traslacionales, que se disminuya la rigidez de las vigas tomando como inercia la correspondiente a la sección fisurada. Por otra parte conviene tener en cuenta, al dimensionar di chas vigas, que han de resistir los momentos adicionales transmitidos por los soportes por el efecto de segundo orden.
1) Como resumen resulta que al aplicar la EH-80 la longitud de pandeo de los soportes pertenecientes a póricos rraslacionales será, normalmente, no menor que 1,2 veces la longitud de los mismos, mientras que aplicando la EH-73 resultaba sólo 0,7 veces esa longitud. Esto liene dos consecuencias:

- Con la EH-73 la inmensa mayoría de los soportes de edilicación cumplian la condición de esbeltez geométrica menor que la limite:

$$
\lambda_{g}=\frac{1}{h} \leqslant 10
$$

y no era necesaria su comprobación a pandeo (en efecto, un soporte de $25 \times 25$ y $3,50 \mathrm{~m}$ de altura la cumplia, por ejemplo). Con la EH-80, por el contrario, muchos soportes de pórticos traslacionales no la cumplen (un soporte de $35 \times 35$ y $3 \mathrm{~m}$ de altura no lo hace), y deben comprobarse.

- La excentricidad adicional de pandeo $e_{a}$ es proporcional al cuadrado de $l_{o}$, Por ello, el aumento de un 70 por 100 en log que se produce rrecuentemente en soportes de pórticos traslacionales, da lugar a un aumento del 200 por 100 de la excentricidad adicional, lo que aumenta bastante la cuantia de armadura necesaria.

En definitiva puede decirse que la EH-80 penaliza uertemente los soportes de póricos Iraslacionales, por lo que será en muchos casos recomendable, desde un punto de vista estrictamente económico, la rigidización de los mismos.

9) Las estructuras de forjados reticulares planos (entrepisos sin vigas) y de vigas planas, si no están fuertemente rigidizadas de modo que puedan considerarse intraslacionales, son especialmente sensibles a los efectos de segun do orden. Al ser muy grande, en estas estructuras, la rigidez de los pllares (sobre lodo los de plantas bajas) frente a las vigas, será muy grande la relación de rigideces $\psi$ y el factor de longitud de pandeo $a$

\section{Valores límites de la esbeltez de piezas. Zonas de pandeo}

La EH-80 mantiene, para soportes aislados, la esbeltez mecánica limite $\lambda_{m}=35$ (a la que corres ponde, para sección rectangular, una esbeltez geométrica $\lambda_{g}=10$ ) dada por la EH-73 como va lor por debajo del cual no es necesaria la compro 
bación a pandeo. Este valor es relativamente alto (el CEB Model Code 78 lo lija en $\lambda_{m}=25 \mathrm{y}$ la Norma DIN $1045-72$ en $\lambda_{m}=201$, pero se ha mantenido para no aumentar demasiado el número de soportes de edificación que deben comprobarse a pandeo.

En la nueva Instrucción aparecen, además, dos nuevas esbelteces limites:

$\lambda_{m}=100$ : limite de aplicabilidad de la fórmula aproximada;

$\lambda_{m}=200$ : esbeltez máxima autorizada por la Ins. trucción.

Como consecuencia, el campo del pandeo de plezas se divide, atendiendo a la esbeltez mecánica, en cuatro zonas claramente delimitadas (fig. 2):

- Zona $0: \lambda_{m} \leqslant 35$

- no hay pandeo; no es necesario tener en cuenta los efectos de segundo orden.

- Zona 1: $35<\lambda_{m} \leqslant 100$

- puede aplicarse la fórmula aproximada (ver apartado 5).

- Zona 2: $100<\lambda_{m} \leqslant 200$

- es preciso hacer un cálculo más riguroso de los electos de segundo orden, pudiendo recurrirse a ábacos o fórmulas (ver apartado 6).

- Zona 3: $200<\lambda_{m}$

- la pieza está fuera de Norma; es precisa una justificación especial de la misma.

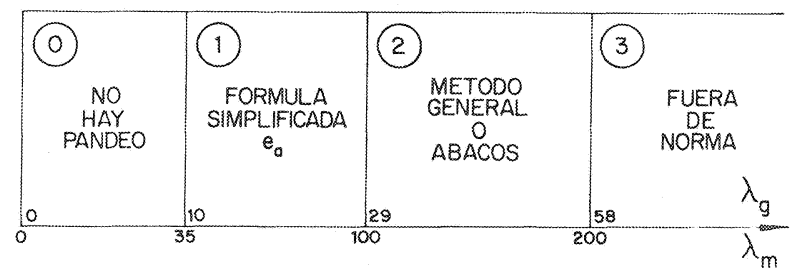

Fig. 2. - Zonas de pandeo según la Instrucción EH-80.

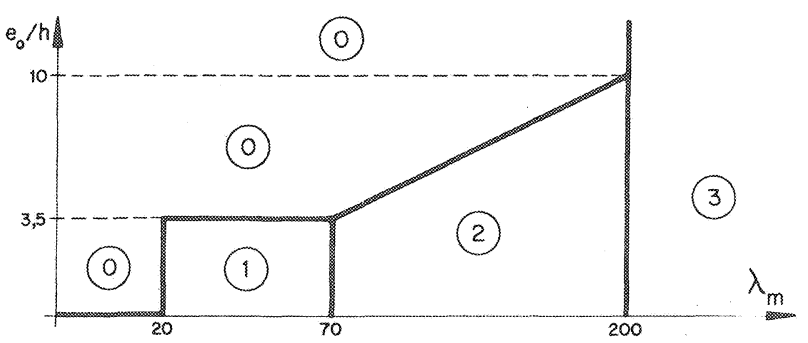

Fig. 3. - Zonas de pandeo según la Norma Alemana DIN 1045-72.
La principal ventaja de esta división del campo de pandeo consiste en permitir, en la gran mayoria de los casos (zonas 0 y 1 ), una resolución rápida y sencilla del problema. Hay que decir, no obstan te, que los limites de las zonas se fijan de forma un tanto arbitraria y simplista, atendiendo única. mente a la esbeltez, que, si bien es el parámetro más importante, no es ciertamente el único que juega en el fenómeno.

La Norma Alemana DIN 1045-72 (5) presenta una división algo más elaborada, atendiendo también a los valores de la excentricidad (fig. 3). De esta forma se elude la comprobación a pandeo de pie zas que puedan estar sometidas a déblles compre siones (por ejemplo, los dinteles de los pórticos).

El tema de los limites de esbeltez está actuámen te en estudio en el seno de la Comisión Permanente III "Pandeo e Inestabilidad" del CEB, existiendo una propuesta italiana que hace intervenir el valor del esfuerzo axil reducido $v, y$ otra francesa que, de acuerdo con la Norma BAEL-80, eli. mina la zona 0 , exigiendo aun para columnas cortas el empleo de un coeficiente de mayoración de acciones adicional que tiene en cuenta los efectos de segundo orden. Este método tiene la ventaja teórica de suprimir el salto brusco que se produce al pasar de la zona 0 a la zona 1.

\section{Valor límite de flexibilidad de pórticos traslacionales. Pandeo global}

Los efectos de segundo orden en un pórtico traslacional (ver apartado 2 b) no quedan cubiertos, en general, por el mero hecho de tener en cuenta una cierta mayoración de momentos al dimensio nar sus soportes (pandeo local o de pieza), ya que en los pórticos esbeltos y flexibles, sometidos a acciones horizontales importantes, pueden producirse grandes desplazamientos laterales $\Delta, y$ la actuación de las cargas verticales $P$ sobre la estructura deformada dará lugar entonces a esfuerzos (axiles, cortantes y momentos) y desplaza. mientos secundarios no lineales, que pueden ser determinantes en el dimensionamiento. Este efecto, conocido como efecto $P-\Delta$, es un efecto de segundo orden que afecta al pórtico considerado en su conjunto (pandeo global o de estructura), y que no era tenido en cuenta por la Instrucción EH-73.

Por ello, la Instrucción EH-80, en su apartado 43.4, establece que para la comprobación de las estructuras traslacionales deberá aplicarse el método general no lineal, esto es, deberá tenerse en cuenta: 
a) La no linealidad geometrica de la estructura, es decir, que las cargas actuan sobre los num dos desplazados y los esfuerzos deben calcum larse teniendo en cuenta la geometria de la estructura deformada.

b) La no linealidad mecánica del material hormigón armado, es decir, que las rigideces de las piezas son variables, ya que dependen de los esfuerzos en las distintas secciones.

Es evidente que la aplicación práctica del cálculo no lineal es complicada, ya que la evaluación de las deformaciones de la estructura y la estimación de las rigideces variables a lo largo de sus plezas es laboriosa y exige el conocimiento previo del armado de la estructura. Resulta asi que ya no es posible separar en dos etapas sucesivas e inde pendientes el cálculo de esfuerzos y el dimensio namiento de secciones, sino que es necesario partir de un armado de la estructura, calcular los esfuerzos y comprobar las secciones, y despues corregir el dimensionamiento si fuera insuficiente o excesivo, en cuyo caso habrá que volver a calcular los esfuerzos, etc. Por otra parte, el cálculo no lineal de esfuerzos en una estructura predefinida no es un proceso directo, sino también iterativo, ya que esfuerzos, rigideces y desplazamientos son magnitudes interdependientes.

Por todo lo anterior el esfuerzo numérico necesario para el cálculo no lineal riguroso de una estructura puede llegar a ser entre 5 y 50 veces mayor que el que requerina el cálculo lineal de primer orden. Fácilmente se comprende la necesidad de utilizar ordenadores de gran capacidad y elevada velocidad de cálculo, y programas ad hoc que aprovechen las caracteristicas especiales de la estructura y del ordenador utilizado.
Para la obtención de las rigideces es preciso partir de un diagrama tension-deformación 10 más realista posible, y válido no sólo para las secciones en agotamiento, que serán sólo unas pocas en toda la estructura, sino para todas las secciones. Por eso la Instrucción EH-80, en el comentario a su apartado 43.2.2, define el diagrama hiperbólico del CEB Model Code 78 (6) (figura 4), preparado de forma que la relación o/ $/ \varepsilon$ para pequeñas deformaciones coincide sensiblemente con el módulo de elasticidad $E_{c m}$ del hormigón, que a su vez es función de la resistencia $f_{c k}$ de éste.

Es importante tener en cuenta que el pandeo es un estado limite último, por 10 cual la comproba. ción de la estructura debe hacerse a partir de las acciones de cálculo (mayoradas) y de los diagra. mas tensióndeformación de cálculo (minorados). Por ello el valor $E_{c m}$ deberá también estar mino. rado. Esto se subraya aqui porque, en este punto, la exposición de la EH-80 podría dar lugar a malas interpretaciones.

Con el fin de obviar la exigencia de comprobar todos los pórticos traslacionales de acuerdo con el método general no lineal, que seria de dificil adopción en la práctica cotidiana, ha parecido necesario incluir unos valores minimos de altura y de flexibilidad para los pórticos de edificación habituales, que tuvieran también en cuenta la importancia de las cargas horizontales, y por debajo de los cuales pueda seguir admitiéndose el cálcum lo usual de primer orden. Esto se hace en el com mentario al apartado 43.4, en el que se establece que para pórticos de edificación de menos de 15 plantas, en los que el desplazamiento máximo en cabeza bajo cargas horizontales características, calculado mediante la teoria de primer orden y con las rigideces correspondientes a las secciones

$$
\begin{aligned}
& \frac{\sigma_{c}}{f_{c d}}=\frac{\eta \mathrm{k}-\eta^{2}}{1+(\mathrm{k}-2) \eta} \\
& \eta=\varepsilon_{c} / \varepsilon_{c 1} \\
& \varepsilon_{c 1}-0,0022 \\
& k=1,1 E_{c m} \varepsilon_{c 1} / f_{c d} \\
& E_{c m}=\text { función de } f_{c k} \text { (TABLA) } \\
& \varepsilon_{c u}=\text { función de } f_{c k} \text { (TABLA) }
\end{aligned}
$$

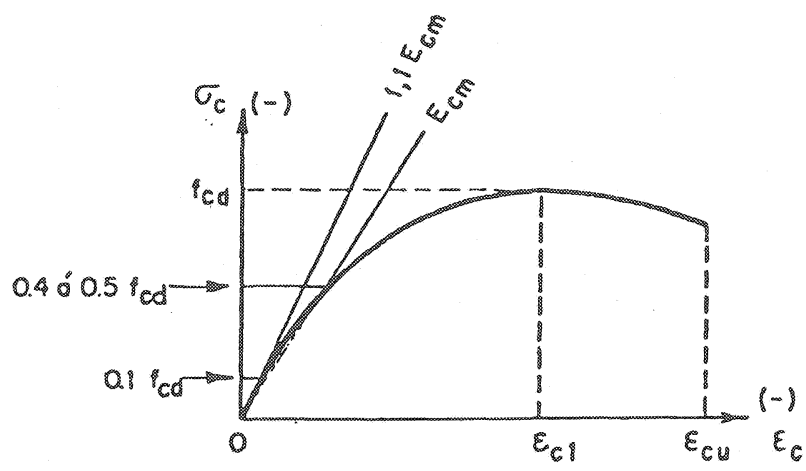

Fig. 4. - Diagrama tensión-deformación hiperbólico para el hormigón.

\begin{tabular}{|c|c|c|c|c|c|c|c|c|c|c|}
\hline$f_{c k}$ & 125 & 150 & 200 & 250 & 300 & 350 & 400 & 450 & 500 & $\mathrm{kp} / \mathrm{cm}^{2}$ \\
\hline$\varepsilon_{c u}$ & $-3,8$ & $-3,7$ & $-3,6$ & $-3,5$ & $-3,4$ & $-3,3$ & $-3,2$ & $-3,1$ & $-3,0$ & $\%$ \\
\hline$E_{c m} \cdot \gamma_{c}$ & 260.000 & 270.000 & 290.000 & 305.000 & 320.000 & 335.000 & 350.000 & 360.000 & 370.000 & $\mathrm{kp} / \mathrm{cm}^{2}$ \\
\hline
\end{tabular}


no fisuradas, no supere $1 / 750$ de la altura total, puede prescindirse del estudio del pórtico en segundo orden, bastando dimensionar cada soporte con los esfuerzos de primer orden mayorados y teniendo en cuenta el pandeo local o de pieza.

Este valor limite de la llexibilidad para pónicos Iraslacionales, original de la Instrucción EH-80, se ha adoptado después de hacer algunos ensayos numéricos en ordenador, que indicaron que para pórticos de edificación regulares cuya altura y fle xibilidad no superen los limites especificados no son de esperar, por el efecto P-A y la no linealldad del material, incrementos en los esfuerzos mayores del 10 al 15 por 100.

En cualquier caso resulta evidente que la Instrucción EH.80 penaliza los pórticos traslacionales esbeltos y flexibles que deban soportar cargas horizontales importantes, obligando an cálculo riguroso y no habitual de los mismos.

En el futuro es de esperar que se generalicen la disponibilidad y la utilización de programas de or denador para el cálculo no lineal de pórticos de edificios, al tiempo que se enuncien nuevos crite rios y métodos simplificados que permitan el tratamiento aproximado de este problema con los programas de cálculo lineal habituales. En ambas direcciones trabaja actualmente, en el Instituto Eduardo Torroja, el Equipo de Investigación «Análisis no lineal de Estructuras", al que pertenecen los autores.

\section{Pandeo en la zona 1. Fórmulas simplificadas}

La Instrucción EH-80 ha conservado, para el cálculo de los momentos de segundo orden en piezas comprimidas de esbeltez reducida $l \lambda_{m} \leqslant 100,20$ na 1) la misma fórmula aproximada ya incluida en la EH-73, y ello porque un estudio numérico de los errores de dicha fórmula en el campo de esbelteces citado, realizado por el segundo autor, demostró que dichos errores quedaban del lado de la seguridad y eran normalmente pequeños, comportándose de hecho la fórmula, en estos dos aspectos, igual o mejor que las fórmulas similares contenidas en otras Normas (ACl 318-77 y DIN 1045-72).

Ello no obstante, se introducen ahora las siguientes precisiones:

a) El campo de aplicación de la fórmula se limita expresamente a soportes de sección constante (hormigón y armaduras) y de esbeltez mecánica no:superior a 100 . b) En el caso, muy frecuente, de soportes con excentricidades de primer orden distintas en sus extremos $\left(e_{02} \neq e_{01}\right)$ y pertenecientes a pórticos intraslacionales, es evidente que la máxima excentricidad de segundo orden $e_{a t}$ situada en un punto a altura intermedia, no coincidirá con la máxima excentricidad de prim mer orden, situada en un extremo, $e_{o 2}$ (fig. 5). Por ello sumar dichas excentricidades sería demasiado conservador. Para tenerlo en cuenta se establecen, para este caso, dos compro baciones:

- a altura intermedia, con una excentricidad de primer orden e. reducida (fig. 5a):

$$
\begin{gathered}
e_{o}=0,6 \cdot e_{o 2}+0,4 \cdot e_{o 1} \& 0,4 \cdot e_{o 2} \\
e_{a}=\begin{array}{c}
\text { función de esta } e_{0} \text { reducida (fómula } \\
\text { aproximada): }
\end{array}
\end{gathered}
$$

- en el extremo, sin excentricidad adicional (fig. $5 b$ ):

$$
\begin{aligned}
& e_{0}=e_{o 2} \\
& e_{a}=0 .
\end{aligned}
$$

\section{Pandeo en la zona 2. Métodos de la Columna Modelo, de la Deformada Senoidal y de las Curvaturas de Referencia}

Para el estudio en segundo orden de soportes de gran esbeltez $\left(\lambda_{m}>100\right)$ o de sección variable no resulta posible la aplicación de la fórmula aproximada del apartado 43.5.2 de la Instrucción EH-80. Deberá, por tanto, recurrirse al método general (cálculo no lineal de segundo orden, apartado 43.2.1 de la EH-80 y apartado 4 de este trabajo).

Teniendo en cuenta no obstante la complejidad, laboriosidad y escasa disponibilidad de dicho mé todo general, y aunque la Instrucción EH-80 no los autorice explicitamente, lo normal será acudir a otros métodos aproximados que hayan demostrado resultados suficientemente buenos en esta zona de pandeo. Entre ellos pueden mencionarse los siguientes:

- Mélodo de la Columna Modelo, expuesto en el Código Modelo del CEB (6) y desarrollado en mayor detalle en el Manual de Pandeo del CEB (7), en el que se incluyen tablas para su aplicación. Estas tablas, de las que se incluye aqui un ejemplo (tabla 1), definen familias de diagramas de interacción para soportes esbeltos, preparados de forma que se incorporan los efectos de segundo orden. 


\section{VALORES DEL MOMENTO REDUCIDO DE PRIMER ORDEN $\mu$}

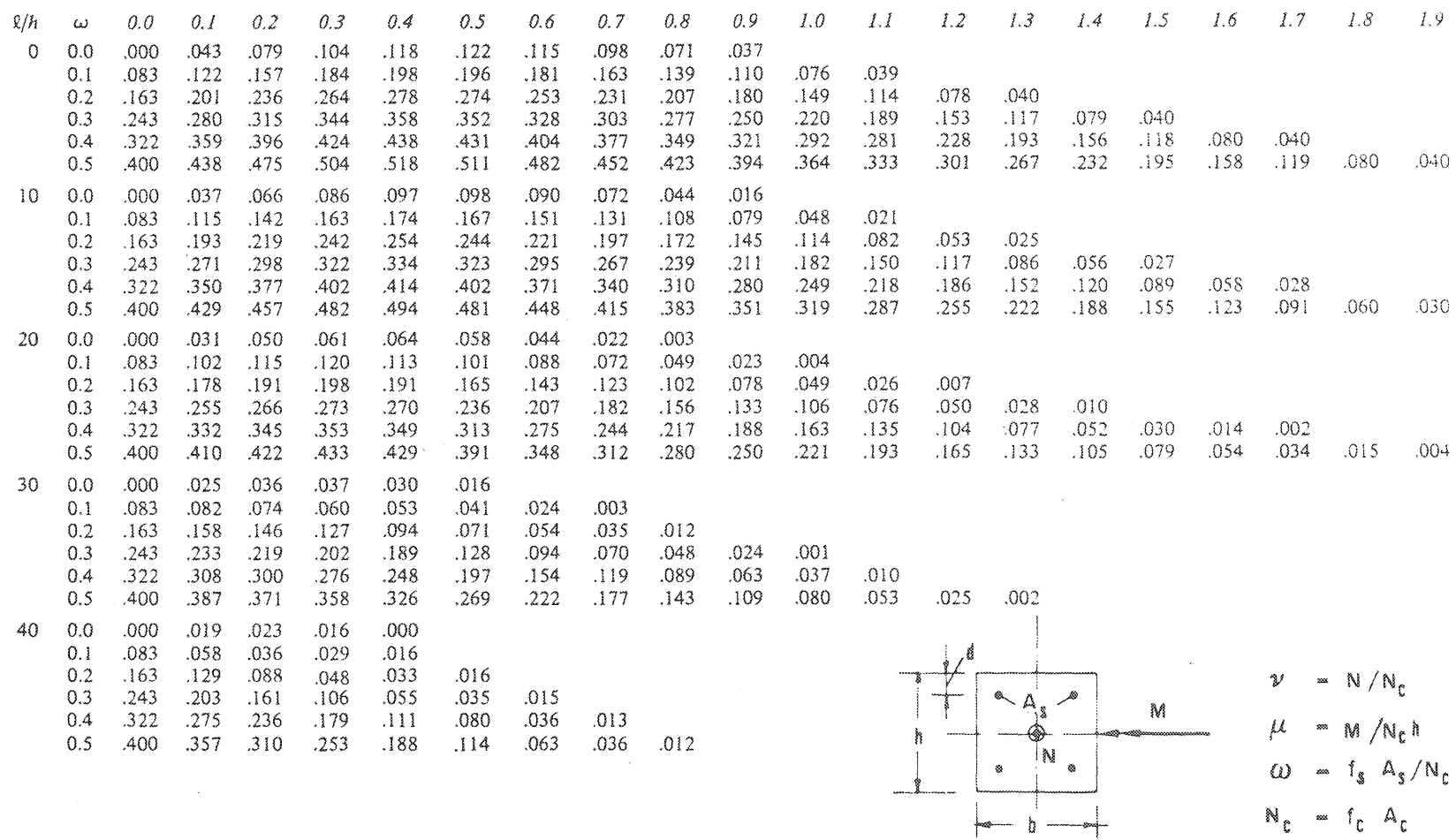

Tabla 1 -Momentos de primer orden relativos $\mu_{1}=M_{2} / N_{\text {h }}$ para soportes esbeltos de sección rectangular con armaduras en las cuatro esquinas. Calculada por el Método de la Columna Modelo para $f_{y d} / E_{s}=0,0020$ y d'/ $=0,10$.

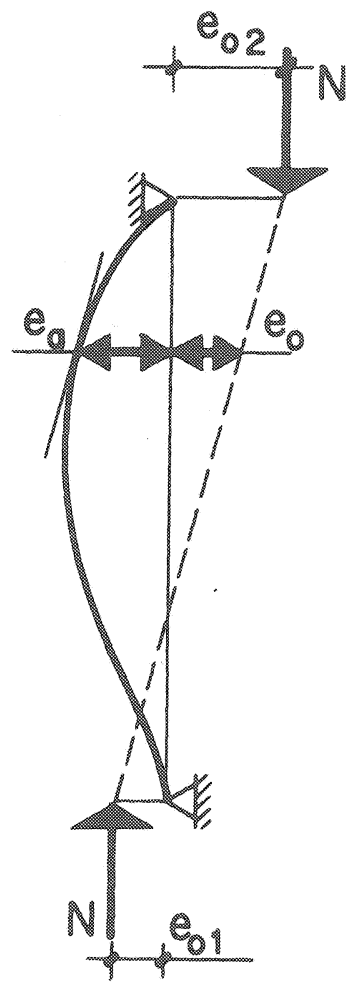

a)

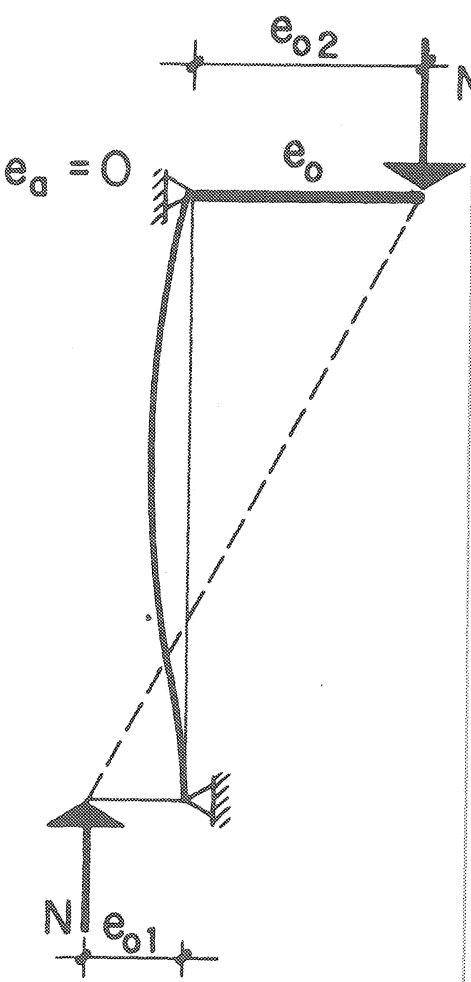

b)

Fig. 5. - Excentricidades inicial $e_{0}$ y adicional $z_{x}$ en ef caso de soportes con excentricidades distintas en sus axtremos.

- Mélodo de la Dolomad Senoidal, expuesto en una reciente publicación de J.J. Arenas (8). Este método es una variante perfeccionada del anterior para incluir excentricidades distintas en los extremos y tener en cuenta la acción de las cargas permanentes (lluencia). En la publicación citada se presenta una colección muy valiosa de diagramas de interacción para la aplicación práctica del método, de los que puede verse uno en la lig. 6.

Mélodo de las Curvaturas de Referencia, original de Hugo Corres (9) (10). Este método con duce al dimensionamiento directo del soporte, esto es, proporciona directamente la cuantía total $\omega$ de armadura necesaria, mediante un proceso gráfico (fig. 7) o aplicando fórmulas aproximadas, cuya precisión es del mismo or den que los dos métodos anteriores, y bastante más elevada que la de la Instrucción EH-80 para esbelteces reducidas. El método ha sido presentado a la Comisión de Pandeo del CEB, que acordo incluirlo en sus próximas publicaclones. 


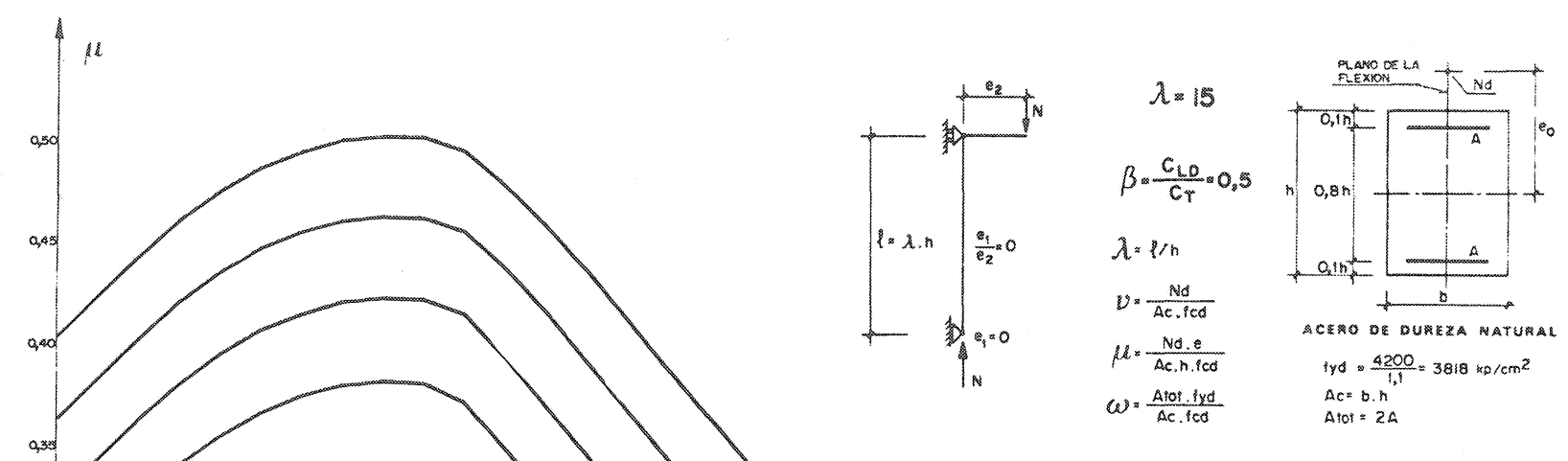

Fig. 6. - Diagramas de interacción para soportes esbeftos con excentricidades dism tintas en los extremos y cargas de larga duración. Calculados por el Método de la Deformada Senoidal.

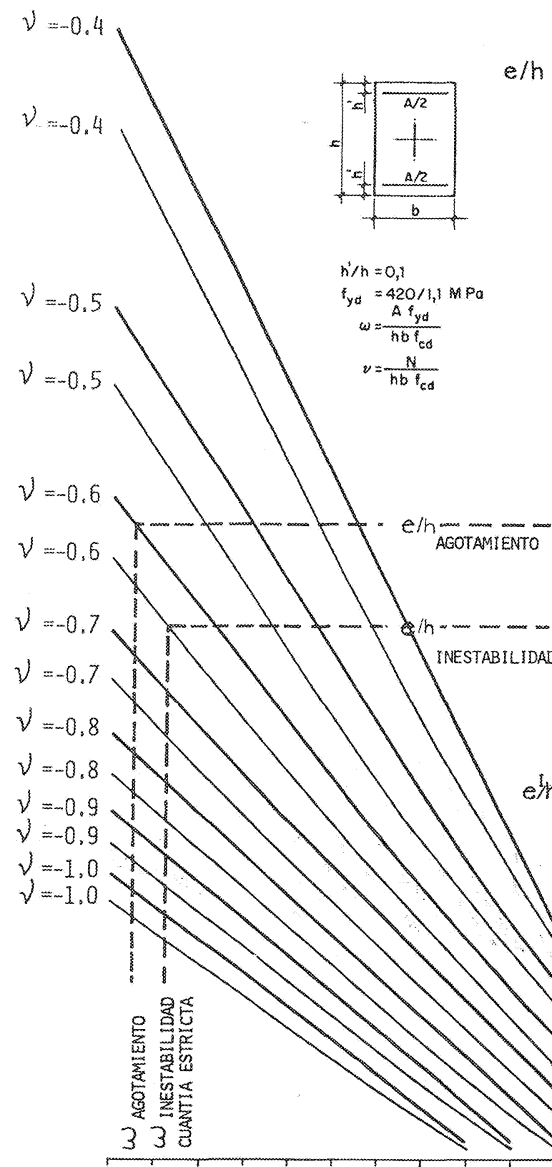

ABACOS DE DIMENSIONAMIENTO $v \leq-0.4$

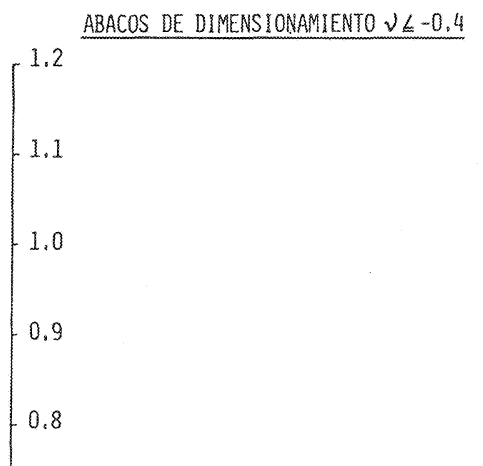




\section{Problemas no cubiertos por la Instrucción EH-80}

Para terminar esta exposición se comentan breve mente dos problemas que no aparecen suficiente mente tratados en la EH.80: el del pandeo bajo cargas sostenidas (fluencia) y el del pandeo en flexión esviada.

En cuanto a la lluencia, su efecto en los problemas de segundo orden es muy importante, ya que produce un aumento de las deformaciones. En el comentario al apartado 43.2.1 la Instrucción EH-80, al exponer las bases del método general, especifim ca que deben tenerse en cuenta los efectos de las deformaciónes diferidas, lo cual puede hacerse estudiando paso a paso la evolución en el tiempo del comportamiento de la estructura, o bien aplicando la simplificación habitual que consiste en partir de un diagrama tensión-deformación para el hormigón que tenga en cuenta las detormaciones a largo plazo.

Si se rrala de una pieza alslada en la zona 1 (35 $\left.<\lambda_{m} \leqslant 100\right)$, es conveniente saber que la Pórmula aproximada dada en el apartado 43.5 .2 de la EH-80 ha sido preparada teniendo en cuenta los efectos de la fluencia, es decir, que si las cargas dominantes son de corta duración queda aprecia. blemente del lado de la seguridad, mientras que para cargas predominantemente permanentes los errores son muy pequeños (11)

Si se trata de una pleza aislada en la zona 2 $\left(100<\lambda_{m}\right)$, ya se ha dicho que los dlagramas de interacción de J.J. Arenas (8) tienen en cuenta la fluencla, para lo cual se presentan diagramas separados para distintos valores de $\beta$, relación de la carga permanente a la carga total. Si se utiliza el Método de la Columna Modelo (7) o el de las Curvaturas de Referencia (9) (10) será preciso incorporar los efectos de la fluencia aumentando debidamente la excentricidad inicial $e_{o ;}$ esto pue de hacerse aplicando las fórmulas dadas en el co mentario al apartado 14.4 .2 del Código Modelo 78 del CEB (6).

En cuanto al pandeo en flexión esviada el tema no se toca en la EH-80. El Código Modelo 78 del CEB incluye, en su apartado 14.5, algunas disposiciones al respecto, pero el Grupo de Trabaio so- bre Pandeo no juzgó oportuno incluirlas en su propuesta de articulado para la Instrucción EH-80 al tener noticias de que recientes investigaciones alemanas parecen demostrar que estas disposicio nes no son correctas. Es necesario estudiar a fondo este dificil problema, que es una de las lineas emprendidas por el Equipo de Investigación sobre "Análisis no lineal de Estructuras" que trabaja actualmente en el Instituto Eduardo Torroja.

\section{Referencias bibliográficas}

1. Johnston, B. C. (Eoitor): "The Column research council guide to design criteria for metal compression members", 2. " Edición. J. Whey, New York, 1966.

2. McGregor, J. G.; Breen, E.: Prrang, E.: "Design of slender concrete columns». ACl Joumal 67, enero, 1970.

3. ACl Committee 318: "Building Code Requirements for Reinforced Concrete (ACl 318-77) $)$. American Concrete Institute, Detroit, 1978.

4. Leonhardt, F.; Mönnig, E.: «Vorlesungen über Massivbau. Erster Tell: Grundlagen zur Bemessung im Stahbetonbauy. Soringer, Berlin, 1973.

5. Deutscher Ausschuss fü Stahbeton: «Beton - und Stahbetonbau. Bemessung und Ausfuhrung (DIN 1045-72). Beton-Kalender, 1976. Wihelm Ernst und Sohn, Berin, 1976.

6. Comité Européen du Béton: "Système Intemational de Régiementation Technique Unifiée des Structures. Code Modele CEB-fIP pour les Structures en Béton). Boletin n. $124 / 125$ Paris, 1978.

7. Comité Européen du Béton: «CEB Design Manual on Buckling». Boletín n. 123 , Paris, 1977

8. Arenas de Pablo, J. J.: "Cálculo de soportes de hormigón armado en teoría de segundo orden». Editores Técnicos Asociados, Barcelona, 1980.

9. Corres, H.: «Dimensionamiento de soportes esbeltos de sección constante de hormigón armado en estado límite último de agotamiento o inestabilidad. Método de las Curvaturas de Referencian. Tesis Doctoral. Escuela Técnica Supe fior de Ingenieros de Caminos. Madrid, 1980.

10. Corres, H.; Morán, F.: «Dimensionamiento de soportes es beltos de hormigón armado. Método de las Curvaturas de Referencia. Grálicos de dimensionamiento. Fórmula de dimensionamiento.) Hormigón y Acero núms. 141 y sgtes. Madrid, 1981 (en prensa).

11. Villegas, 1.: "Estudio comparativo de la inestabilidad de soportes rectangulares de hormigón armado según la Instrucción EH 73, el Código ACl y los ábacos de J.J. Arenas». Hormigón y Acero n. 137, Madrid, 1980. 